\title{
Postoperative executive function in adult moyamoya disease: a preliminary study of its functional anatomy and behavioral correlates
}

\author{
${ }^{*} Y u$ Lei, MD, ${ }^{1}$ Yan-Jiang Li, MD,, Qi-Hao Guo, MD, PhD, ${ }^{2}$ Xing-Dang Liu, MD, PhD, ${ }^{3}$ \\ Zhuang Liu, MD, ${ }^{3}$ Wei Ni, MD, PhD, ${ }^{1}$ Jia-Bin Su, MD, ${ }^{1}$ Heng Yang, MD, ${ }^{1}$ Han-Qiang Jiang, MD, PhD, ${ }^{1}$ \\ Bin Xu, MD, PhD, ${ }^{1}$ Yu-Xiang Gu, MD, PhD, ${ }^{1}$ and Ying Mao, MD, PhD ${ }^{1}$
}

Departments of ${ }^{1}$ Neurosurgery, ${ }^{2}$ Neurology, and ${ }^{3}$ Nuclear Medicine, Huashan Hospital, Fudan University, Shanghai, China

OBJECTIVE Chronic frontal hemodynamic disturbances are associated with executive dysfunction in adult patients with moyamoya disease (MMD). However, the impact of surgical revascularization on executive dysfunction and its underlying mechanism remains unclear. The aim of the present study was to examine the postoperative radiological correlates of cognitive improvement and thereby explore its underlying mechanism.

METHODS Fourteen patients who met the inclusion criteria were identified at Huashan Hospital, were operated on, and were successfully followed up for 6 months. Postoperative changes in cortical perfusion and regional amplitude of lowfrequency fluctuations (ALFF) were examined by SPECT and resting-state functional MRI, respectively. Executive function was evaluated by 2 tests (Trail Making Test Part B and the summation of executive subtests of Memory and Executive Screening [MES-EX]). Follow-up neuropsychological outcomes were then correlated with radiological changes to identify nodes functioning as leading contributors to postoperative executive outcomes.

RESULTS All patients underwent successful unilateral bypass procedures, with some operations performed on the left side and some on the right side. At the 6-month follow-up, the baseline and follow-up test scores for the different sides did not differ significantly. The group with good collaterals (Matsushima Grade A, 9 patients) exhibited significantly increased postoperative perfusion (change in $[\triangle$ ] hemodynamics) in bilateral frontal (left, $p=0.009$; right, $p=0.003$ ) and left parietal lobe $(p=0.014)$. The Spearman's correlation test suggested that only the right frontal lobe exhibited significant positive postoperative radiological correlates with cognitive performance ( $\triangle$ MES-EX vs $\triangle$ hemodynamics, $r=0.620, p=0.018 ; \triangle$ MES-EX vs $\triangle$ ALFF, $r=0.676, p=0.008 ; \triangle$ hemodynamics vs $\triangle A L F F, r=0.547, p=0.043)$. Subsequent regional ALFF analysis revealed that the right dorsolateral prefrontal cortex (DLPFC) was the only node in the responsible hemisphere to exhibit significant postoperative changes.

CONCLUSIONS The results not only advance our understanding of pathological interactions of postoperative executive performance in adult MMD, but also indicate that the right DLPFC amplitude might be a quantitative predictor of postoperative executive control improvement.

http://thejns.org/doi/abs/10.3171/2015.12.JNS151499

KEY WORDS moyamoya disease; executive function; perfusion; resting-state fMRI; surgical revascularization; vascular disorders

ABBREVIATIONS ALFF = amplitude of low-frequency fluctuation; DLPFC = dorsolateral prefrontal cortex; DMN = default mode network; $E C N=$ executive-control network; EDMS = encephaloduromyosynangiosis; $\mathrm{fMRI}$ = functional MRI; MES-EX = summation of executive subtests of Memory and Executive Screening; MMD = moyamoya disease; $\mathrm{PCC}=$ posterior cingulate cortex; $\mathrm{PCU}=$ precuneus; $\mathrm{ROI}=$ region of interest; $\mathrm{STA}-\mathrm{MCA}=$ superficial temporal artery-middle cerebral artery; TIA = transient ischemic attack; TMT-B = Trail Making Test Part $B ; \triangle=$ change in.

SUBMITTED June 28, 2015. ACCEPTED December 29, 2015.

INCLUDE WHEN CITING Published online April 8, 2016; DOI: 10.3171/2015.12.JNS151499.

* Drs. Lei and Li contributed equally to this work. 
$\mathrm{M}$ OYAMOYa disease (MMD) is characterized by progressive stenosis of the terminal portion of the bilateral internal carotid arteries and is associated with an extensive network of cerebral collaterals of unknown etiologies. ${ }^{22}$ MMD in adult patients is commonly accompanied by vascular cognitive impairment, with executive dysfunction being the hallmark cognitive deficit. ${ }^{10,26}$ Previous studies have shown that surgical revascularization is the only effective form of treatment for adult MMD, ${ }^{19,26}$ but its effectiveness in cognitive protection is still disputed.

Previous studies have revealed that vascular cognitive impairment is the consequence of chronic deficiencies in regional cerebral blood flow. ${ }^{3,6}$ Furthermore, structural changes in the brain have been detected in response to hemodynamic deficiencies, and surgical revascularization has been reported to reverse such structural changes. ${ }^{7}$ The impact of surgery on executive performance in adult MMD and the underlying mechanism has not, however, been well studied.

Executive function arises from a collection of brain areas acting together as networks, particularly the executive control network (ECN), comprising mainly the dorsolateral prefrontal cortex (DLPFC) and posterior parietal cortex. ${ }^{16,25}$ A few studies of resting-state functional MRI (fMRI) have focused on the neuroanatomical deficits of the ECN in adult MMD, ${ }^{13,15}$ but to the best of our knowledge, the impact of surgical revascularization on these networks has not been reported yet. The amplitude of lowfrequency fluctuations (ALFF) $(<0.1 \mathrm{~Hz})$ in blood oxygen level-dependent fMRI during rest arises primarily from spontaneous fluctuations of brain physiology and neuronal activity, which provides insight into the intrinsic functional architecture of the brain. ${ }^{2}$ Thus far, ALFF analysis has been widely applied to detect spatial patterns in the brain activity of patients with cognitive impairment in fMRI studies. ${ }^{15,24}$ Using this resting-state fMRI approach, the present study was carried out to investigate the functional correlates of executive performance in patients undergoing surgery to explore the underlying mechanism and to identify regions that may serve as markers to detect cognitive changes in the procedure.

\section{Methods}

\section{Participants and Intervention}

The observational study was approved by the institutional review board of Huashan Hospital and was conducted in accordance with the Declaration of Helsinki. All participants provided informed consent. Inclusion criteria were 1) right-handed Chinese population aged 18-80 years; 2) no evidence of infarct in the cerebral cortex, basal ganglia, brainstem, or cerebellum, but small patches of hyperintense signal neither larger than the arbitrary cutoff of $8 \mathrm{~mm}$ in maximum dimension on T2-weighted MR images nor cystic in the cerebral subcortical white matter could be involved; ${ }^{12} 3$ ) no evidence of intracerebral hemorrhage; 4) diagnosis through digital subtraction angiography; 5) no surgery before recruitment; 6) physically capable of cognitive evaluation; and 7) absence of significant psychiatric disorders or neurological diseases that could compromise cognition. Patients with severe systemic or other cerebrovascular diseases and those taking drugs, such as benzodiazepine clonazepam, were excluded. ${ }^{14}$ Fourteen consecutive adult patients (case group, mean age at diagnosis $37.4 \pm 7.7$ years, $57.1 \%$ male) were identified at our center from July 2013 to June 2014.

Surgical revascularization at our center was achieved by a combined superficial temporal artery-middle cerebral artery (STA-MCA) bypass and encephaloduromyosynangiosis (EDMS), ${ }^{11,30}$ and hemispheres with transient ischemic attacks (TIAs) matching the hemodynamic defects were targeted. Bypass patency was assessed intraoperatively through indocyanine green fluorescence imaging or Doppler ultrasound. Angiographic follow-up was evaluated by 2 radiologists who were not involved in the surgery. Transdural collaterals after revascularization were evaluated in accordance with the criteria proposed by Matsushima and Inaba, ${ }^{18}$ defined as Grade A (area perfused by the synangiosis greater than two-thirds of the MCA territory), Grade B (one-third to approximately two-thirds), and Grade $\mathrm{C}$ (less than one-third).

After having been screened by MR angiography, 10 healthy, young subjects (mean age at assessment $39.2 \pm$ 6.8 years, $60.0 \%$ male), without mental diseases, memory complaints, or any cerebrovascular disease, were successfully included in the study as controls.

\section{Neuropsychological Assessment}

A comprehensive battery of neuropsychological tests, including memory, attention, language, executive function, and visuospatial ability, was adopted, among which the Trail Making Test Part B (TMT-B) and a summation of executive subtests of Memory and Executive Screening (MES-EX) were adopted to assess the executive function preoperatively and during follow-up (Appendix 1). TMT$\mathrm{B}$ is a popular test for identifying executive dysfunction, but the floor effect limits its application in people with a low level of education. ${ }^{17,29}$ However, the MES-EX is a brief screening test that is sensitive for illiterate and poorly educated individuals and focuses on 2 common components of executive function: set shift and dominant inhibition. ${ }^{9}$

According to the previously established criterion based on performance deviation from the published normative means of the 2 tests, patients scoring 1.5 SDs away from the normative means of the 2 tests were considered to have domain impairments. ${ }^{8,14}$ In the present study, executive dysfunction was defined as impairments in both tests (scoring criteria shown in Appendix 2). A trained rater, unaware of the study aim or the patients' diagnoses, administered the neuropsychological tests to the participants.

\section{Evaluation of Hemodynamic Changes}

Perfusion values in the brain regions were evaluated using SPECT/CT (Siemens, Symbia T16). Patients were maintained in the supine position, with eyes closed in a dark and quiet room for 30 minutes, and SPECT/CT scans were obtained after the intravenous injection of $1480 \mathrm{MBq}$ of ${ }^{99 \mathrm{~m} T c-e t h y l ~ c y s t e i n a t e ~ d i m e r ~(N e u r o l i t e, ~ B r i s t o l-M y e r s ~}$ Squibb Pharma). Data were collected into a $128 \times 128$ matrix through a $360^{\circ}$ rotation at steps of $2.8^{\circ}$ for 80,000 
counts per view. Filtered back-projection using an OSEM (ordered subset expectation materialization) ${ }^{8,16}$ was used for SPECT/CT image reconstruction. Each brain perfusion SPECT/CT study was quantified and compared with an age- and sex-matched normal database using the NeuroGam software package (Segami Corporation). This software applies an affine anatomical coregistration through blocks of data defined in the Talairach space.

Perfusion values were calculated for participants' brain regions, and those values were expressed in terms of SDs from the age- and sex-adjusted mean value derived from the normal database. Abnormal areas with hemodynamic defects were defined as those with decreased uptake below 2 SDs of the normal mean. To allow for determination of postoperative hemodynamic improvement, patients were required to undergo SPECT/CT preoperatively and during follow-up. An experienced nuclear medical physician, blinded to the results of neuropsychological testing and patient diagnosis, performed the image analysis.

\section{Resting-State fMRI Acquisition and Preprocessing}

Data were acquired using a 3.0-Tesla intraoperative MRI system (Siemens Medical Solutions). ${ }^{15}$ All fMRI data were acquired using gradient echo-planar imaging (TR 2000 msec, TE 35 msec; FOV $240 \times 240 \mathrm{~mm}^{2}$; matrix $64 \times 64$ $\mathrm{mm}$; slice thickness $4 \mathrm{~mm}$ ). The duration of the scan was approximately 10 minutes. For structural images, a 1-mmthick axial section was achieved using the fast spoiled gradient recalled echo inversion recovery sequence, and the parameters were as follows: TR $1000 \mathrm{msec}$, TE $5 \mathrm{msec}$, TI $400 \mathrm{msec}$, flip angle $20^{\circ}$, interslice space $0 \mathrm{~mm}$, FOV 240 $\times 240 \mathrm{~mm}^{2}$, acquisition matrix $256 \times 256$.

Statistical parametric mapping (SPM8, http://www.fil. ion.ucl.ac.uk/spm/) was adopted to preprocess the blood oxygen level-dependent data. Functional volumes were realigned using least-squares minimization without higherorder corrections for spin history and then normalized to the Montreal Neurological Institute template. Images were then resampled to $3-\mathrm{mm}$ isotropic voxels and smoothed with a 4-mm full-width at half-maximum Gaussian kernel. Then the REST (Resting-State fMRI Data Analysis Toolkit) was adopted to perform linear trend subtraction and temporal filtering $(0.01-0.08 \mathrm{~Hz})$ on the time series of each voxel, aiming at reducing the effect of low-frequency drifts and high-frequency noise. ${ }^{2}$ Finally, to lower the impact of variability across subjects (patients and controls) enrolled, we divided the ALFF value of each voxel by the global mean ALFF within the whole-brain mask achieved previously.

We assigned experienced technicians who were unaware of the study aim or the patient diagnoses to acquire the images and preprocess the data.

\section{Data Processing}

\section{Postoperative Radiological Correlates of Neuropsychological Outcomes}

Subtraction measure was used to define postoperative improvement, expressed as $\triangle \mathrm{ALFF}, \triangle$ hemodynamics, and $\triangle$ MES-EX $\left(\triangle\right.$ value $=$ value $_{\text {post }}-$ value $\left._{\text {pre }}\right)$. Regions of interest (ROIs) for ALFF were indicated by the SPECT/
CT map overlaid on the cortical surface, including bilateral frontal and parietal lobes. Mean ALFF and perfusion values in each ROI were then extracted, both preoperatively and during follow-up. Subsequently, Spearman's correlation test was adopted to measure correlates between $\triangle \mathrm{ALFF}, \triangle$ hemodynamics, and $\triangle \mathrm{MES}$-EX separately in each ROI, to determine which hemisphere was directly responsible for postoperative cognitive improvement.

\section{Regional ALFF Analysis}

To elucidate the functional anatomy of postoperative cognitive changes and further explore the underlying mechanism, regional ALFF was analyzed. A 2-sample t-test was performed primarily between the case and control groups on individual normalized ALFF maps, obtaining the abnormal brain regions with ALFF changes of adult MMD (cluster size $>2295 \mathrm{~mm}^{3}$, corrected $\mathrm{p}<$ 0.05). The correction was determined by Monte Carlo simulations with the Analysis of Functional Neuroimages (AFNI) AlphaSim software. The linear correlation was then calculated between MES-EX scores and mean ALFF values across all voxels in these abnormal regions in the case group (corrected $\mathrm{p}<0.05$ ). After identifying the correlated regions, a mask was created to confine the regions for postoperative analysis.

Subsequently, a paired-sample t-test was performed between the post- and preoperative regional ALFF under the mask in the case group (results were generated automatically by the software, corrected $\mathrm{p}<0.05$ ). The brain nodes that were generated were identified using a published atlas of ROIs (http://findlab.stanford.edu/functional_ROIs. $\mathrm{html}){ }^{27}$ Finally, results were referred back to the previously responsible hemisphere to identify nodes functioning as leading contributors to postoperative cognitive improvement.

\section{Statistics}

Database management and statistical analyses were performed via SPSS 16.0 software (SPSS Inc.). Continuous variables were expressed as percentages or the means \pm SD. The Mann-Whitney U-test was used to examine the differences between the case and control groups, as the variables had an abnormal distribution. Spearman's correlation analysis was used to examine the correlation between the neuropsychological and radiological data. A significance level of 0.05 was assumed.

\section{Results}

\section{Patient Characteristics}

The case and control groups did not significantly differ in age $(p=0.565)$, education level $(p=0.345)$, or sex distribution $(\mathrm{p}=0.611)$. The case group spent significantly more time completing the TMT-B $(148.5 \pm 56.5$ vs 102.7 \pm 31.3 seconds, $\mathrm{z}=2.235, \mathrm{p}=0.025$ ) and exhibited a lower mean score on the MES-EX $(40.8 \pm 8.9$ vs $47.0 \pm 4.7$, z $=2.563, \mathrm{p}=0.010)$ than the controls. The time for TMT$\mathrm{B}$ completion and the MES-EX scores correlated well (r $=-0.821 ; \mathrm{p}=0.001)$. One patient $(7.1 \%)$ could not complete the TMT-B due to illiteracy; thus, we adopted the MES-EX as the main measurement for further analysis. 


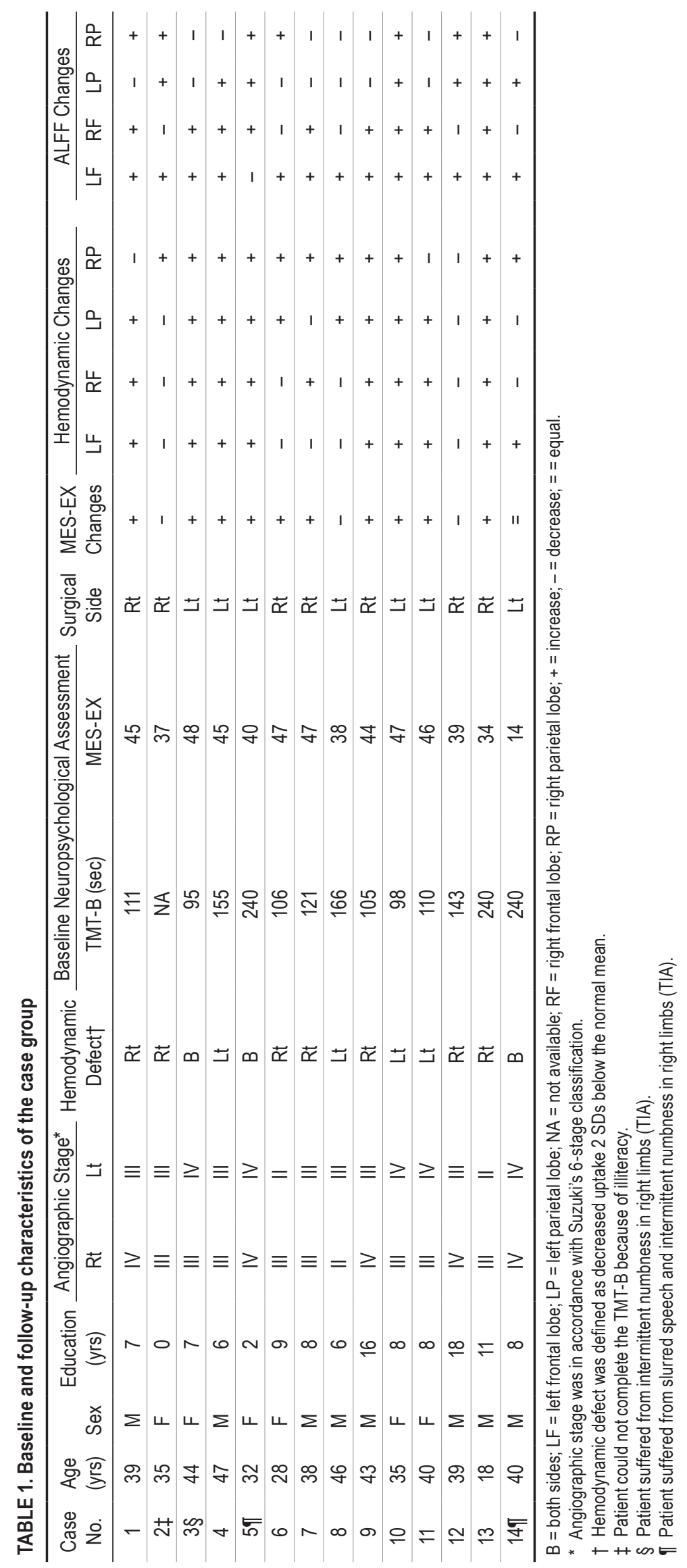


The characteristics of the patients are listed in Table 1. According to Suzuki's 6-stage classification, ${ }^{23} 3$ hemispheres of the case group were graded Stage II, 15 were Stage III, and the remaining 10 hemispheres were Stage IV.

All patients underwent a unilateral bypass (of the left side in 7 patients), and surgeries were successfully performed, with a $100 \%$ patency rate for each anastomotic stoma procedure. No patients experienced ischemic or hemorrhagic episodes during the 6-month follow-up. The results of neuropsychological follow-ups are shown in Fig. 1. With regard to different operated on sides, patients did not differ significantly in either baseline MES-EX ( $p=$ $0.671)$ or $\triangle$ MES-EX $(p=0.930)$. Angiographic follow-up indicated that 9 patients were Grade $\mathrm{A}$ according to the Matsushima criteria, whereas 4 were Grade B and 1 was Grade C.

\section{Postoperative Radiological Correlates of Neuropsychological Outcomes}

The characteristics of neuropsychological and radiological follow-up of the patients are listed in Table 1. ROIs in the current study were set as bilateral frontal and parietal lobes. To assess the impact of bypass on postoperative hemodynamic changes, patients were divided into 2 groups: groups with good (Grade A) and moderate (Grades B+C) collaterals. The Mann-Whitney U-test was then used to account for differences in variance between the 2 groups. The results indicated that the group with good collaterals exhibited significantly increased $\triangle$ hemodynamics in the left frontal lobe $(p=0.009)$, right frontal lobe $(p=0.003)$, and left parietal lobe $(\mathrm{p}=0.014)$ and also trended toward increased $\triangle$ hemodynamics in the right parietal lobe $(\mathrm{p}=$ 0.205; Fig. 2).

The results of the Spearman's correlation test suggested that only the right frontal lobe exhibited significant positive correlations between $\triangle$ hemodynamics, $\triangle \mathrm{ALFF}$, and $\triangle$ MES-EX, separately $(\triangle \mathrm{MES}$-EX vs $\triangle$ hemodynamics, $\mathrm{r}=0.620, \mathrm{p}=0.018 ; \triangle \mathrm{MES}-\mathrm{EX}$ vs $\triangle \mathrm{ALFF}, \mathrm{r}=0.676$, $\mathrm{p}=0.008 ; \triangle$ hemodynamics vs $\triangle \mathrm{ALFF}, \mathrm{r}=0.547, \mathrm{p}=$ $0.043)$. In addition, $\triangle$ hemodynamics of the left frontal lobe showed a significant positive correlation with $\triangle$ MES-EX $(r=0.610, p=0.020)$ but a negative correlation with $\triangle \mathrm{ALFF}(\mathrm{r}=-0.566, \mathrm{p}=0.035)$. The left parietal lobe exhibited only a significant correlation between $\triangle$ hemodynamics and $\triangle$ MES-EX $(r=0.589, p=0.027$; Fig. 3, details of the correlation are shown in Appendix 3). Thus, the right frontal lobe was deemed to be the etiologically responsible hemisphere for postoperative cognitive improvement.

\section{Regional ALFF Analysis}

The ALFF patterns of the case group are presented in Fig. 4A. Visual inspection revealed that, compared with controls, significant decreases in ALFF were observed mainly in the frontal and parietal lobes, whereas some prefrontal areas simultaneously exhibited significant ALFF increases. Subsequently, we performed a linear correlation test between the MES-EX scores and the mean ALFF values across all voxels in these abnormal regions (Fig. 4B). Significant positive correlations were mainly observed in the parietal lobe, including the bilateral inferior parietal cortex, the posterior cingulate cortex (PCC), and the precuneus $(\mathrm{PCu})$, as well as the anterior prefrontal cortex. Additionally, significant negative correlations were detected in the frontal lobe, including the bilateral DLPFC and subcortical regions.

The following paired-sample t-test indicated that, within the generated regions, only the $\mathrm{PCC}$, left $\mathrm{PCu}$, and right DLPFC exhibited significant postoperative ALFF differences (Table 2 and Fig. 5). Notably, the right DLPFC was the only node detected in the right frontal lobe. Thus, we concluded that the right DLPFC might be the primary
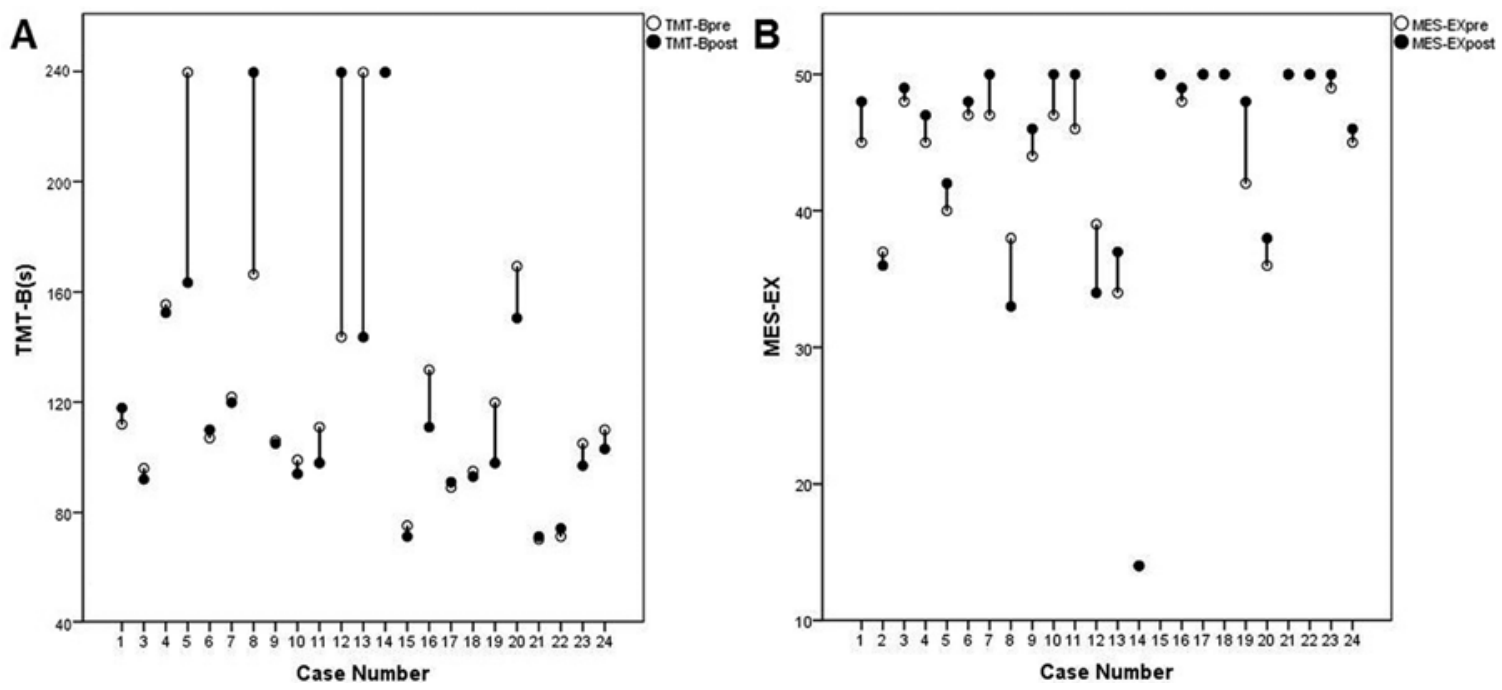

FIG. 1. Graphs showing the results of baseline and follow-up TMT-B (A) and MES-EX (B) of the case and control groups. Participants 1-14 represent patients in the case group, whereas participants 15-24 represent the healthy controls. The patient in Case 2 could not complete the TMT-B because of illiteracy. TMT- pre/post $_{\text {i }}=$ baseline/follow-up time to complete the TMT-B; MES-EX pre/post $=$ baseline/follow-up MES-EX scores; $(\mathrm{s})$ = seconds. 


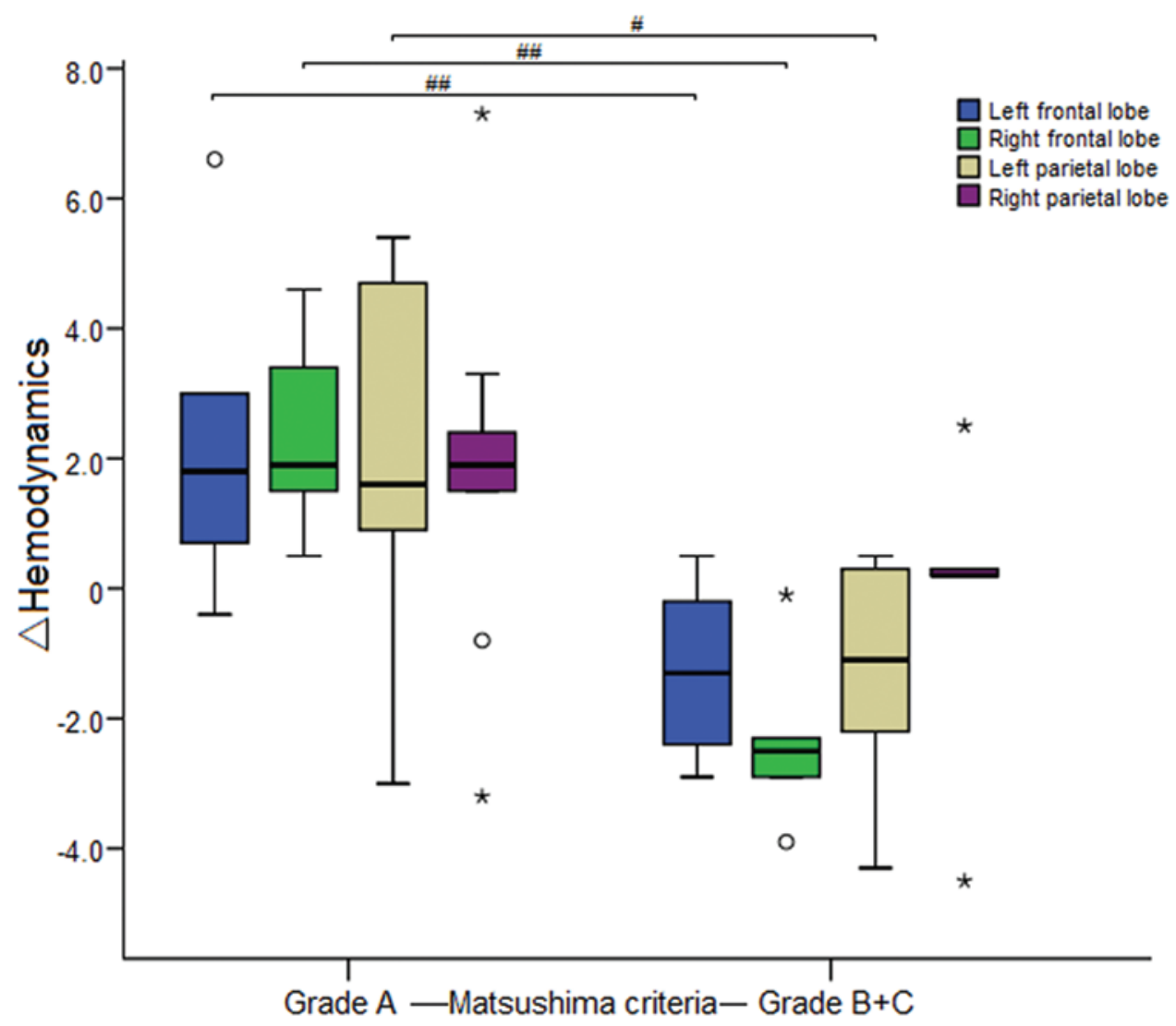

FIG. 2. Box plot showing differences in $\triangle$ hemodynamics between patients with good (Grade $A$ ) and moderate (Grades $B+C)$ collaterals in bilateral frontal and parietal lobes (Mann-Whitney U-test). The box plots show the full range (whiskers), interquartile range, and median values. ${ }^{\circ}=$ Abnormal value $>2$ SDs of the normal mean; ${ }^{*}=$ abnormal value $>$ SD of the normal mean; \# $=p<$ 0.05 ; $\# \#=p<0.01$. Figure is available in color online only.

node for determining postoperative executive control improvement, whereas the PCC and left PCu followed with activity autoregulation due to topological reorganization.

\section{Discussion}

Using SPECT and resting-state fMRI, we investigated the impact of postoperative hemodynamic changes on executive function and the underlying pathophysiology in adult MMD. The main findings of the present study were as follows: 1) after intracranial bypass surgery, only in the right frontal lobe were the changes of regional hemodynamics simultaneously consistent with measures of intrinsic brain activity and cognitive performance; 2) the right DLPFC amplitude might serve as a quantitative predictor of postoperative executive control improvement; and 3) the PCC and left PCu were also involved in topological reorganization of brain networks associated with postoperative executive performance.

Although frontal hemodynamic deficits have been demonstrated to cause executive dysfunction in adult MMD patients,, 34 it is unclear whether restoring frontal perfusion by surgical revascularization can arrest or reverse this dysfunction. Moreover, the fundamental mechanism underlying this phenomenon is also unclear. The present study primarily confirmed the positive correlation between the postoperative perfusion improvement and surgical revascularization. Interestingly, the correlation was observed in all the frontal regions but in only a few of the parietal regions. We supposed that the main cause for this might have been due to the surgical strategy. All patients in the study were offered a unilateral combined STA-MCA bypass and EDMS via the modified pterional approach. To supply blood flow over a wide surface of the brain, we performed the bypass surgery though a frontotemporal crani-

TABLE 2. Brain regions showing significant postoperative ALFF changes in the case group*

\begin{tabular}{lrrrrr}
\hline & & \multicolumn{3}{c}{ MNI Coordinates $(\mathrm{mm})$} & \\
\cline { 3 - 5 } Brain Region & Vol $\left(\mathrm{mm}^{3}\right)$ & \multicolumn{1}{c}{$\mathrm{X}$} & $\mathrm{Y}$ & $\mathrm{Z}$ & Max T Value† \\
\hline Lt PCu & 2025 & -12 & -51 & 9 & 4.448 \\
\hline Lt PCC & 459 & -6 & -42 & 9 & 4.274 \\
\hline Rt PCC & 162 & 9 & -36 & 12 & 3.577 \\
\hline Rt DLPFC & 648 & 30 & 36 & 45 & 2.976 \\
\hline
\end{tabular}

$\mathrm{MNI}=$ Montreal Neurological Institute.

* For clusters with multiple anatomical locations, only the peaks of activations are labeled.

$\dagger$ T-statistical value of peak voxel showing significant post- versus preoperative ALFF changes in the case group (cluster size $>2295 \mathrm{~mm}^{3}$, corrected $p<$ 0.05). 

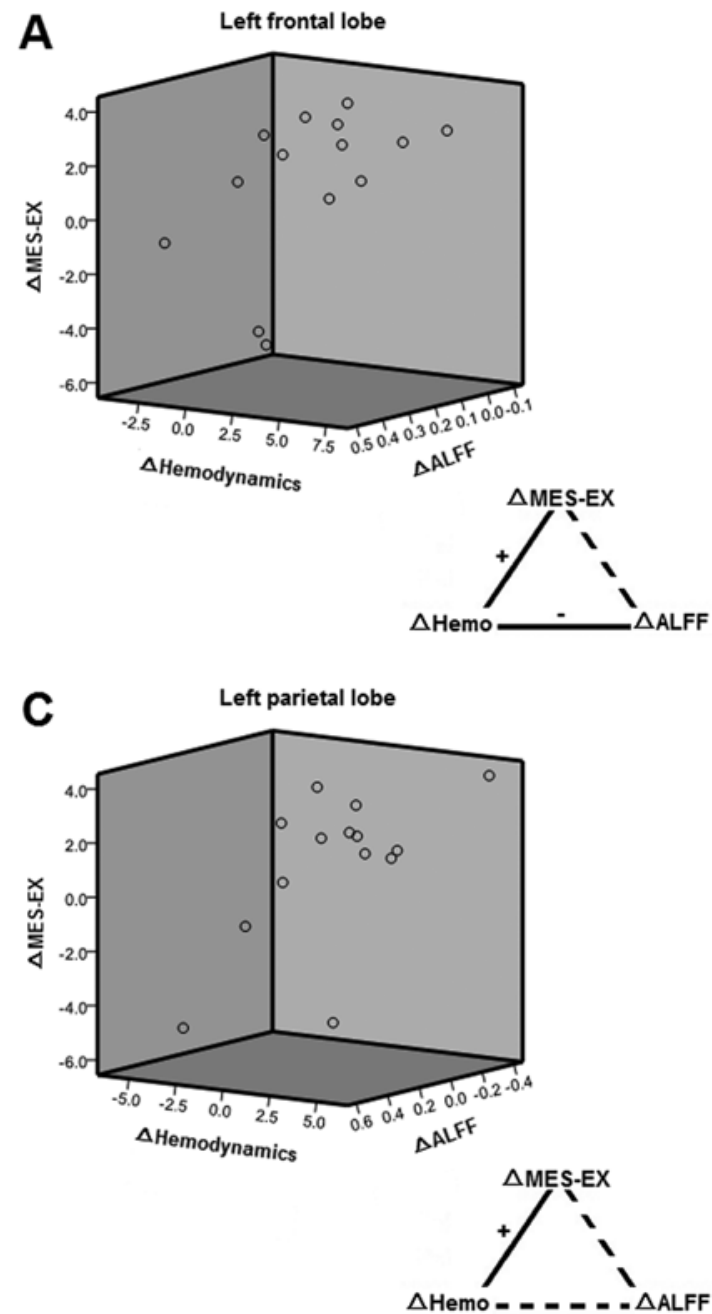
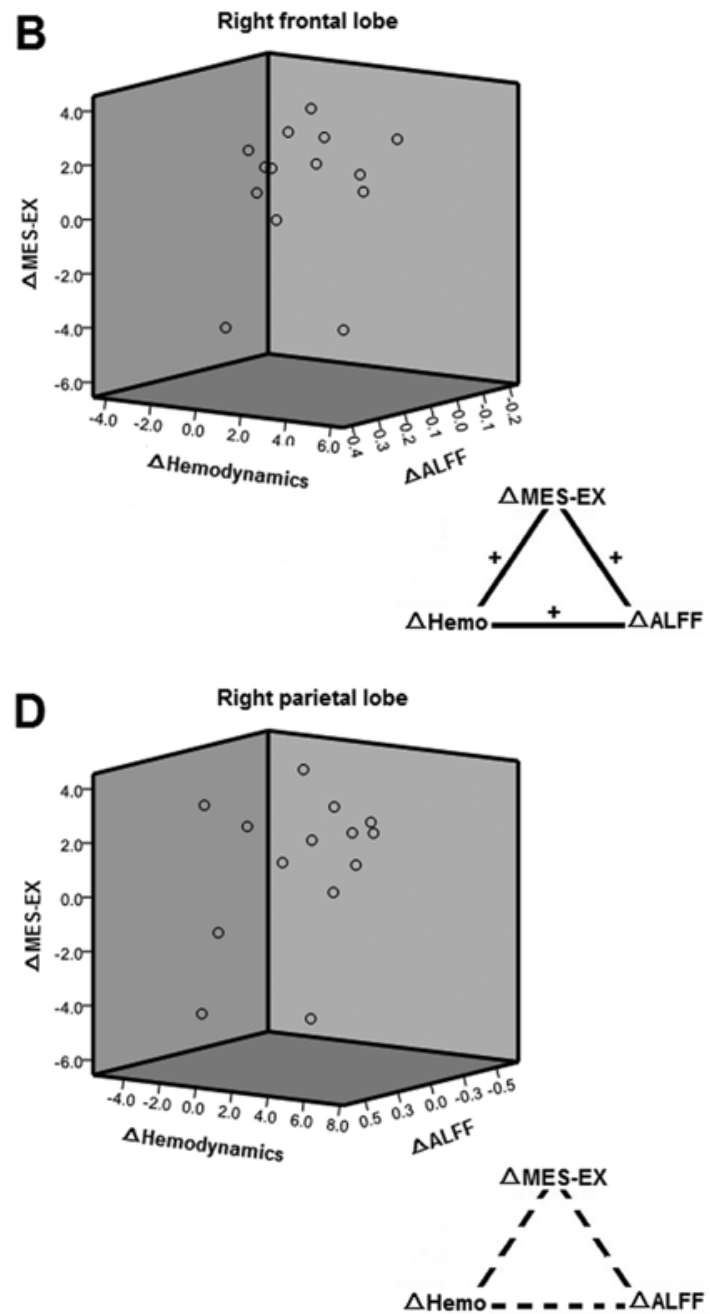

FIG. 3. 3D scatter diagram showing the correlates between $\triangle A L F F, \triangle$ hemodynamics, and $\triangle M E S$-EX separately in the left frontal (A), right frontal (B), left parietal (C), and right parietal (D) lobes. The solid lines indicate significant correlation, and the dashed lines indicate insignificant correlation. Hemo = hemodynamics; + = positive correlation; - = negative correlation.

otomy that covered large areas of the frontal and temporal lobes rather than the parietal lobe. For the STA-MCA bypass, 1 branch of the STA was anastomosed to M4 or distal branches of the MCA, which mainly augmented blood flow in the frontal and temporal lobes. Additionally, EDMS has been proved to be effective in improving the hemodynamics of the frontal lobe. ${ }^{4}$ Thus, compared with the parietal regions, it is reasonable to notice more robust hemodynamic changes in frontal regions.

We subsequently noted that, although postoperative cognitive improvement occurred in response to bilateral frontal perfusion restoration, only the right frontal lobe exhibited a simultaneous correlation with regional ALFF changes. This result implies that the etiologically responsible brain nodes might be located in the right frontal lobe. In addition, we noted that an increase in regional perfusion did not always occur in the revascularized hemisphere, a phenomenon reported in a previous study. ${ }^{7}$ Variations in vascular collateral blood flow might be the cause of this phenomenon, and further investigation is needed.

Executive function is spatially driven by a set of net- works that operate together, such as the left ECN, right ECN, and the dorsal default mode network (DMN). ${ }^{16,25,28}$ Weiland et al. ${ }^{25}$ indicated that the right DLPFC and left superior parietal gyrus/angular gyrus independently play a key role in bilateral ECNs separately. Wu et al. ${ }^{27}$ also found that the right DLPFC and anterior cingulate cortex were the only 2 hubs in the bilateral ECNs that showed significant functional connectivity strength with executive function. The DLPFC is deemed to be a key node in the regulation of a wide range of executive processes, such as strategy formation, set shifting, and working memory. ${ }^{20,21}$ Taken together, we hypothesized that amplitude changes in the right DLPFC might serve as a biomarker for executive performance after surgery in adult MMD.

In addition, the $\mathrm{PCC}$ and left $\mathrm{PCu}$ were found to exhibit significant ALFF changes after surgery. The $\mathrm{PCC} / \mathrm{PCu}$ is a core component of the DMN, implying that the DMN also contributed to postoperative changes of executive performance. ${ }^{1,5,25}$ Previous studies indicate that the ECN and DMN operate together to support executive function. ${ }^{16,28}$ However, to our knowledge, few studies have related the 

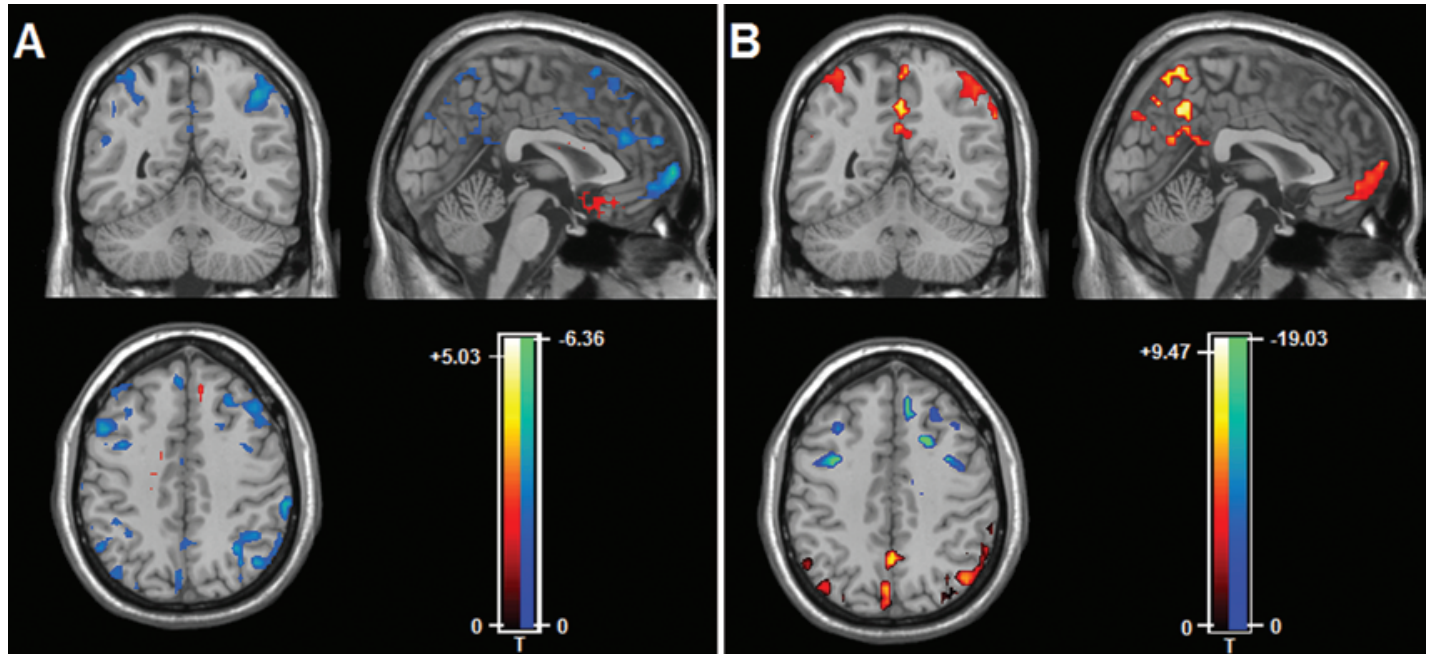

FIG. 4. T-statistical and correlation maps showing the difference between the case and control groups. A: T-statistical difference maps showing that compared with controls, significant decreases in ALFF values were observed mainly in the frontal and parietal lobes, whereas some prefrontal areas simultaneously exhibited significant ALFF value increases. B: Correlation maps of MES-EX score and ALFF within the abnormal regions showing that significant positive correlations are mainly observed in the parietal lobe, such as in the bilateral inferior parietal cortex, the PCC, and the PCu, as well as in the anterior prefrontal cortex. However, significant negative correlations are detected in the frontal lobe, including the bilateral DLPFC and subcortical regions. The statistical threshold was set at corrected $p<0.05$. Figure is available in color online only.

DMN and ECN to postoperative executive function in adult patients with MMD. Thus, more studies are required for validation of the present result.

Notably, there are certain limitations to the present study. Primarily, due to the strict inclusion and exclusion criteria, patients with cerebral infarction or intracranial hemorrhage were not included, resulting in a patient popu- lation with a relatively mild executive dysfunction. Thus, we cannot rule out the possibility that as adult MMD progresses, the ALFF values of nodes mentioned in the present study may change in the opposite direction or new nodes may be identified as being involved in cognitive changes. Second, the present findings are based on a small sample size. In the case group, 1 patient could not

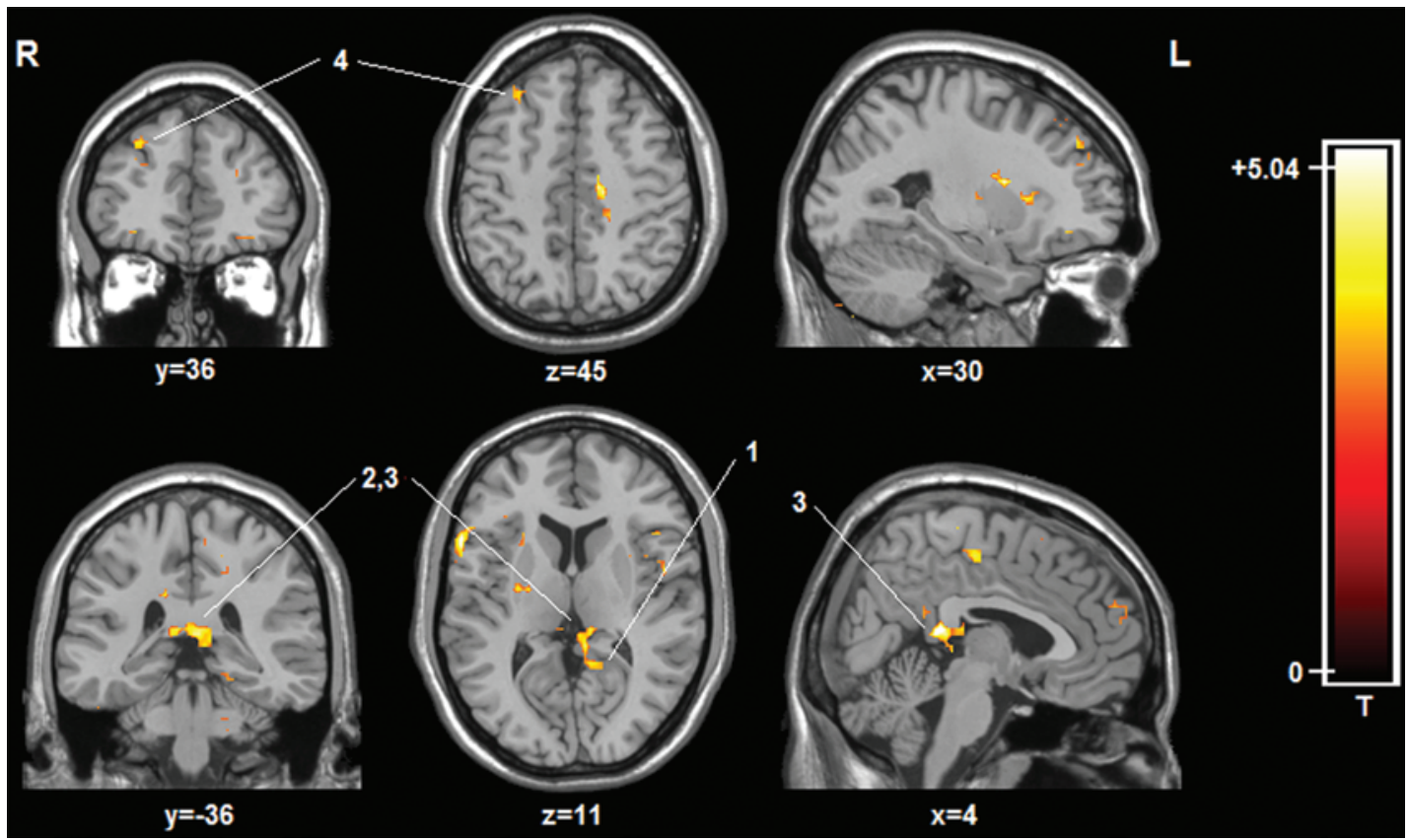

FIG. 5. T-statistical maps showing the results of a paired-sample t-test between post- and preoperative ALFF values. Within the mask generated in Fig. 4B, only the left PCu (indicated by 1), the PCC (2 and 3), and the right DLPFC (4) exhibited significantly different ALFF values. The statistical threshold was set at corrected $p<0.05 . L=$ left; $R=$ right. Figure is available in color online only. 
complete the TMT-B. Considering the small sample size, ruling out any patient would weaken the statistical power; therefore, we adopted the MES-EX as the main measurement and proved its sensitivity for all patients in the cohort. We propose that further investigations with increased cohort size are needed to boost the statistical power of the results. In addition, it was difficult to keep the results blinded because of the study design and easy recognition of patients with surgical scars. Although several measures were taken to keep results relatively objective, the potential for bias remained.

\section{Conclusions}

The results of the present study not only suggest that the right DLPFC is the key node of executive control performance after surgical revascularization in adult MMD but also advance our understanding of postoperative functional network reorganization. In future studies, we will explore the functional connectivity between brain networks related to postoperative executive function in adult MMD.

\section{Acknowledgments}

This study was supported by grant 2014CB541604 from the National Key Basic Research Program of China (973 Program) and grant 81371307 from the National Natural Science Foundation of China. We thank Dr. Ping He for his excellent assistance in statistical analysis in this paper.

\section{References}

1. Baggio HC, Segura B, Sala-Llonch R, Marti MJ, Valldeoriola F, Compta Y, et al: Cognitive impairment and resting-state network connectivity in Parkinson's disease. Hum Brain Mapp 36:199-212, 2015

2. Biswal B, Yetkin FZ, Haughton VM, Hyde JS: Functional connectivity in the motor cortex of resting human brain using echo-planar MRI. Magn Reson Med 34:537-541, 1995

3. Calviere L, Catalaa I, Marlats F, Viguier A, Bonneville F, Cognard C, et al: Correlation between cognitive impairment and cerebral hemodynamic disturbances on perfusion magnetic resonance imaging in European adults with moyamoya disease. Clinical article. J Neurosurg 113:753-759, 2010

4. Calviere L, Ssi Yan Kai G, Catalaa I, Marlats F, Bonneville $\mathrm{F}$, Larrue V: Executive dysfunction in adults with moyamoya disease is associated with increased diffusion in frontal white matter. J Neurol Neurosurg Psychiatry 83:591-593, 2012

5. Dai ZJ, Yan CG, Li KC, Wang ZQ, Wang JH, Cao M, et al: Identifying and mapping connectivity patterns of brain network hubs in Alzheimer's disease. Cereb Cortex 25:37233742,2015

6. Festa JR, Schwarz LR, Pliskin N, Cullum CM, Lacritz L, Charbel FT, et al: Neurocognitive dysfunction in adult moyamoya disease. J Neurol 257:806-815, 2010

7. Fierstra J, Maclean DB, Fisher JA, Han JS, Mandell DM, Conklin J, et al: Surgical revascularization reverses cerebral cortical thinning in patients with severe cerebrovascular steno-occlusive disease. Stroke 42:1631-1637, 2011

8. Gorelick PB, Scuteri A, Black SE, Decarli C, Greenberg SM, Iadecola $\mathrm{C}$, et al: Vascular contributions to cognitive impairment and dementia: a statement for healthcare professionals from the American Heart Association/American Stroke Association. Stroke 42:2672-2713, 2011

9. Guo QH, Zhou B, Zhao QH, Wang B, Hong Z: Memory and Executive Screening (MES): a brief cognitive test for detecting mild cognitive impairment. BMC Neurol 12:119, 2012
10. Hachinski V, Iadecola C, Petersen RC, Breteler MM, Nyenhuis DL, Black SE, et al: National Institute of Neurological Disorders and Stroke-Canadian Stroke Network vascular cognitive impairment harmonization standards. Stroke 37:2220-2241, 2006 (Erratum in Stroke 38:1118, 2007)

11. Jiang H, Ni W, Xu B, Lei Y, Tian Y, Xu F, et al: Outcome in adult patients with hemorrhagic moyamoya disease after combined extracranial-intracranial bypass. J Neurosurg 121:1048-1055, 2014

12. Karzmark P, Zeifert PD, Bell-Stephens TE, Steinberg GK, Dorfman LJ: Neurocognitive impairment in adults with moyamoya disease without stroke. Neurosurgery 70:634-638, 2012

13. Kazumata K, Tha KK, Narita H, Kusumi I, Shichinohe H, Ito $\mathrm{M}$, et al: Chronic ischemia alters brain microstructural integrity and cognitive performance in adult moyamoya disease. Stroke 46:354-360, 2015

14. Lei Y, Guo Q, Li Y, Jiang H, Ni W, Gu Y: [Characteristics of cognitive impairment in adults with cerebral ischemia.] Zhonghua Yi Xue Za Zhi 94:984-989, 2014 (Chinese)

15. Lei Y, Li Y, Ni W, Jiang H, Yang Z, Guo Q, et al: Spontaneous brain activity in adult patients with moyamoya disease: a resting-state fMRI study. Brain Res 1546:27-33, 2014

16. Liang X, Zou QH, He Y, Yang YH: Topologically reorganized connectivity architecture of default-mode, executivecontrol, and salience networks across working memory task loads. Cereb Cortex 26:1501-1511, 2016

17. Lu L, Bigler ED: Normative data on trail making test for neurologically normal, Chinese-speaking adults. Appl Neuropsychol 9:219-225, 2002

18. Matsushima Y, Inaba Y: Moyamoya disease in children and its surgical treatment. Introduction of a new surgical procedure and its follow-up angiograms. Childs Brain 11:155170, 1984

19. Pandey P, Steinberg GK: Neurosurgical advances in the treatment of moyamoya disease. Stroke 42:3304-3310, 2011

20. Pettit LD, Bastin ME, Smith C, Bak TH, Gillingwater TH, Abrahams S: Executive deficits, not processing speed relates to abnormalities in distinct prefrontal tracts in amyotrophic lateral sclerosis. Brain 136:3290-3304, 2013

21. Royall DR, Lauterbach EC, Cummings JL, Reeve A, Rummans TA, Kaufer DI, et al: Executive control function: a review of its promise and challenges for clinical research. J Neuropsychiatry Clin Neurosci 14:377-405, 2002

22. Suzuki J, Kodama N: Moyamoya disease-a review. Stroke 14:104-109, 1983

23. Suzuki J, Takaku A: Cerebrovascular "moyamoya" disease. Disease showing abnormal net-like vessels in base of brain. Arch Neurol 20:288-299, 1969

24. Wang Z, Yan C, Zhao C, Qi Z, Zhou W, Lu J, et al: Spatial patterns of intrinsic brain activity in mild cognitive impairment and Alzheimer's disease: a resting-state functional MRI study. Hum Brain Mapp 32:1720-1740, 2011

25. Weiland BJ, Sabbineni A, Calhoun VD, Welsh RC, Hutchison KE: Reduced executive and default network functional connectivity in cigarette smokers. Hum Brain Mapp 36:872882,2015

26. Weinberg DG, Rahme RJ, Aoun SG, Batjer HH, Bendok BR: Moyamoya disease: functional and neurocognitive outcomes in the pediatric and adult populations. Neurosurg Focus 30(6):E21, 2011

27. Wu L, Soder RB, Schoemaker D, Carbonnell F, Sziklas V, Rowley J, et al: Resting state executive control network adaptations in amnestic mild cognitive impairment. J Alzheimers Dis 40:993-1004, 2014

28. Xin F, Lei X: Competition between frontoparietal control and default networks supports social working memory and empathy. Soc Cogn Affect Neurosci 10:1144-1152, 2015

29. Zhao Q, Guo Q, Li F, Zhou Y, Wang B, Hong Z: The Shape 
Trail Test: application of a new variant of the Trail Making Test. PLoS One 8:e57333, 2013

30. Zhu FP, Zhang Y, Higurashi M, Xu B, Gu YX, Mao Y, et al: Haemodynamic analysis of vessel remodelling in STA-MCA bypass for moyamoya disease and its impact on bypass patency. J Biomech 47:1800-1805, 2014

\section{Disclosures}

The authors report no conflict of interest concerning the materials or methods used in this study or the findings specified in this paper.

\section{Author Contributions}

Conception and design: Gu, Guo, X Liu, Mao. Acquisition of data: Z Liu, Ni, Yang, Jiang. Analysis and interpretation of data: Lei, Li, Su. Drafting the article: Lei. Critically revising the article: Gu, Lei, Li, Guo, X Liu, Z Liu, Ni, Su, Jiang, Xu, Mao. Reviewed submitted version of manuscript: Gu, Lei, Li, Guo, X $\mathrm{Liu}, \mathrm{Z} \mathrm{Liu}, \mathrm{Ni}$, Su, Jiang, Xu, Mao. Approved the final version of the manuscript on behalf of all authors: Gu. Statistical analysis: Lei. Administrative/technical/material support: Xu. Study supervision: Guo, Mao.

\section{Supplemental Information}

\section{Online-Only Content}

Supplemental material is available with the online version of the article.

Appendices 1-3. http://thejns.org/doi/suppl/10.3171/2015.12. JNS151499.

\section{Previous Presentations}

Portions of this paper were presented in 2015 at the 4th International Moyamoya Meeting (July 2-4, Berlin, Germany).

\section{Correspondence}

Yu-Xiang Gu, Department of Neurosurgery, Huashan Hospital of Fudan University, 12\# Wulumuqi Zhong Rd., Shanghai 200040, China. email: guyuxiang1972@126.com. 